\title{
Property of seamless immersed boundary method for large eddy simulation of incompressible turbulent flows
}

\author{
Kyohei TAJIRI*, Hidetoshi NISHIDA** and Mitsuru TANAKA** \\ * Department of Mechanical and System Engineering, Kyoto Institute of Technology \\ Matsugasaki, Sakyo-ku, Kyoto 606-8585, Japan \\ E-mail: d2821002@edu.kit.ac.jp \\ ** Department of Mechanical and System Engineering, Kyoto Institute of Technology \\ Matsugasaki, Sakyo-ku, Kyoto 606-8585, Japan
}

Received 12 March 2014

\begin{abstract}
In this paper, the simulations of incompressible turbulent flow are presented by applying the seamless immersed boundary method (SIBM) to the large eddy simulation (LES) on the Cartesian grid. The static Smagorinsky model with the wall damping function is adopted as modeling the subgrid-scale (SGS) component in the present approach. In order to validate the SIBM for LES, the turbulent channel flow which is one of the most fundamental wall turbulent flow is considered with $R e_{\tau}=180$ defined by the friction velocity. In comparison with the reference DNS and LES data, the present result is closer to the DNS data than the LES one. Moreover, the present SIBM results are in good agreement with the results of conventional approach with wall boundary condition. Secondly, the numerical simulations of flow around a sphere which is a basic shape with curvilinear boundary are carried out with the Reynolds number $R e=3700$ and 10000 where the flow around a sphere is the turbulent flow. As a result, it is found that the present results are in very good agreement with the reference LES ones with the dynamic SGS model. Then, it is concluded that the present SIBM is very promising for the LES of incompressible turbulent flows.
\end{abstract}

Key words : Computational fluid dynamics, Computational method, Immersed boundary method, Turbulent flow, Large eddy simulation

\section{Introduction}

In the flow analysis, there are mainly two ways, i.e., the boundary fitted coordinates (BFC) and the Cartesian grid approaches. Conventionally, the BFC approach is usually chosen for the flow around objects, because of its more accurate results. However, the huge computational time for the grid generation is sometimes required in the case with more complicated shape. Therefore, the Cartesian grid approach is spotlighted again, due to its easy grid generation and high computational efficiency. In the Cartesian grid approach, it is not necessary to newly generate the computational grid, even if the complicated objects are included in the computational domain. Only the boundaries of objects have to be specified. Consequently, the computational time for the grid generation can be reduced significantly. Moreover, the automation of computational codes, such as the grid generation, becomes very easy.

In the Cartesian grid approach, the immersed boundary method (IBM) (Peskin and McQueen, 1989) is conventionally used. In order to satisfy the velocity condition on the boundary, e.g., the non-slip condition, the IBM requires the additional forcing term in the momentum equations. The additional forcing term is determined by the feedback forcing (Goldstein, et al., 1993) or direct forcing (Fadlun, et al., 2000). Today, the direct forcing is dominant, because the feedback forcing has the severe time step restriction than the direct forcing. Also, the original direct forcing provides the unphysical oscillations of the pressure near the boundary. In order to improve this weak point, the seamless immersed boundary method (SIBM) (Nishida and Sasao, 2006) is recently proposed, so that the unphysical oscillations of the pressure near the boundary can be removed and the precise pressure distribution can be determined on the boundary. In the past, the SIBM has been applied to the various laminar flows (Nishida and Tajiri, 2009) (Nishida and Tajiri, 2010) (Tajiri, et al, 2013). However, 
the property of SIBM for the turbulent flow has not been investigated yet. Then, the property of SIBM for the turbulent flow by using the large eddy simulation (LES) is discussed in this paper.

First, the LES of turbulent plane channel flow is considered. The result obtained by the SIBM with simple static Smagorinsky subgrid-scale (SGS) model is compared with the result obtained by the original IBM, the conventional LES result with wall boundary condition, and the reference results, in order to validate the present SIBM. Finally, the LESs of turbulent flow around a sphere with $R e=3700$ and 10000 are performed by using the SIBM. Then, the property of SIBM for the turbulent flow is discussed.

\section{Seamless Immersed Boundary Method}

\subsection{Governing equations for LES}

The filtered continuity equation and incompressible Navier-Stokes equations are written as,

$$
\begin{aligned}
& \frac{\partial \bar{u}_{i}}{\partial x_{i}}=0, \\
& \frac{\partial \bar{u}_{i}}{\partial t}=\frac{\partial \bar{F}_{i j}}{\partial x_{j}}+\bar{G}_{i}, \\
& \bar{F}_{i j}=-\delta_{i j} \bar{p}-\bar{u}_{i} \bar{u}_{j}+\frac{1}{R e}\left(\frac{\partial \bar{u}_{i}}{\partial x_{j}}+\frac{\partial \bar{u}_{j}}{\partial x_{i}}\right)-{\overline{u_{i}^{\prime} u_{j}^{\prime}}}^{(S G S)},
\end{aligned}
$$

where, $\bar{u}_{i}$ and $\bar{p}$ are the velocity component and the pressure in the grid scale (GS). $\bar{G}_{i}$ in the momentum equations denotes the filtered additional forcing term for IBM and SIBM. ${\overline{u_{i}^{\prime} u_{j}^{\prime}}}^{(S G S)}$ is the Reynolds stress of the SGS component defined by

$$
-{\overline{u_{i}^{\prime} u_{j}^{\prime}}}^{(S G S)}=v_{S G S}\left(\frac{\partial \bar{u}_{i}}{\partial x_{j}}+\frac{\partial \bar{u}_{j}}{\partial x_{i}}\right)-\frac{1}{3} \delta_{i j}{\overline{u_{k}^{\prime} u_{k}^{\prime}}}^{(S G S)} .
$$

The static Smagorinsky model with the wall damping function $f_{s}$ is adopted as modeling the SGS component. The static Smagorinsky model is written by

$$
\begin{aligned}
& v_{S G S}=\left(C_{s} f_{s} \Delta s\right)^{2}\left(2 \bar{S}_{i j} \bar{S}_{i j}\right)^{1 / 2}, \\
& f_{s}=1-\exp \left(-r^{+} / 25\right), \\
& \bar{S}_{i j} \equiv\left(\partial \bar{u}_{i} / \partial x_{j}+\partial \bar{u}_{j} / \partial x_{i}\right) / 2, \\
& \Delta s=\sqrt[3]{\Delta x \Delta y \Delta z},
\end{aligned}
$$

where $c_{s}$ is the Smagorinsky model constant. In this paper, it is set as 0.1 . Moreover, the superscript + shows the normalized value by the friction velocity which is defined by the average wall shear stress on the wall. $r^{+}$denotes the shortest distance from the surface of a wall. By applying these models, Eq.(3) is rewritten by

$$
\begin{aligned}
& \bar{F}_{i j}=-\delta_{i j} P-\bar{u}_{i} \bar{u}_{j}+\left(\frac{1}{R e}+v_{S G S}\right)\left(\frac{\partial \bar{u}_{i}}{\partial x_{j}}+\frac{\partial \bar{u}_{j}}{\partial x_{i}}\right), \\
& P=\bar{p}+\frac{1}{3} \delta_{i j}{\overline{u_{k}^{\prime} u_{k}^{\prime}}}^{(S G S)} .
\end{aligned}
$$

\subsection{Seamless immersed boundary method}

In order to adopt IBM or SIBM, the additional forcing term in the momentum equations, $G_{i}$, should be estimated. There are mainly two ways, i.e., the feedback (Goldstein, et al., 1993) (Saiki and Biringen, 1996) and direct (Fadlun, et al., 2000) forcing term estimations. In this paper, the direct forcing term estimation is adopted in accordance with the previous studies (Nishida and Sasao, 2006) (Nishida and Tajiri, 2009) (Nishida and Tajiri, 2010) (Tajiri, et al, 2013).

The direct forcing term estimation is shown in Fig. 1(a). We explain in 2-D but the extension to 3-D is straightforward. For the forward Euler time integration, the forcing term can be determined by

$$
\bar{G}_{i}^{n}=-\frac{\partial \bar{F}_{i j}^{n}}{\partial x_{j}}+\frac{\bar{U}_{i}^{n+1}-\bar{u}_{i}^{n}}{\Delta t},
$$


where $\bar{U}_{i}^{n+1}$ denotes the interpolated velocity by linear interpolation. Namely, the external force is specified as the velocity components at next time step satisfy the relation, $u_{i}^{\overline{n+1}}=\bar{U}_{i}^{n+1}$. In the forcing term estimation of the conventional IBM, the grid points added forcing term are restricted near the boundary only. In this approach, the non-negligible velocity appears inside the boundary. Also, the pressure distributions near the boundary show the unphysical oscillations because of the pressure jump. In order to remove the unphysical oscillations near the boundary, the SIBM was proposed (Nishida and Sasao, 2006). In the SIBM, the forcing term is added not only on the grid points near the boundary but also in the region inside the boundary shown in Fig. 1(b). In the region inside the boundary, the forcing term is determined by satisfying the relation, $\bar{U}_{i}^{n+1}=\bar{U}_{b}$, where $\bar{U}_{b}$ is the specified velocity, e.g., $\bar{U}_{b}=0$ in the stationary solid media.

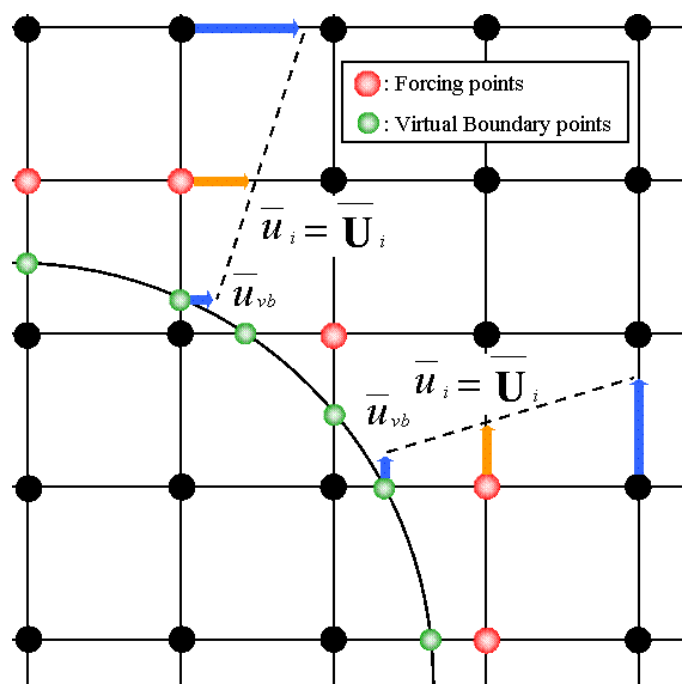

(a) Conventional IBM.

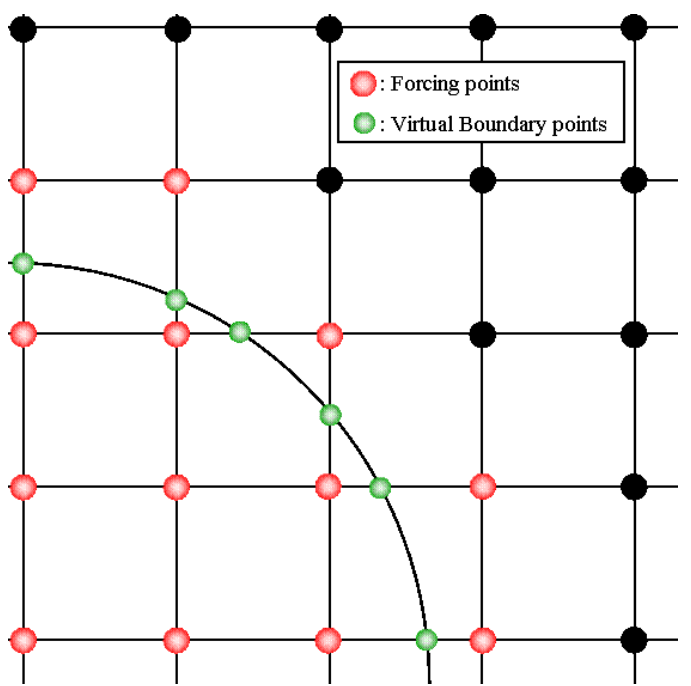

(b) Seamless IBM.

Fig. 1 Grid points added forcing and heat flux terms.

\subsection{Numerical method}

The incompressible Navier-Stokes equations (2) are solved by the second order finite difference method on the collocated grid arrangement. The convective terms are discretized by the second order fully conservative finite difference method (Morinishi, et al., 1998). The diffusion and pressure terms are discretized by the conventional second order centered finite difference method. For the time integration, the fractional step approach (Rhie and Chow, 1983) based on the two-step Runge-Kutta scheme (Renaut, 1990) is applied. For the incompressible Navier-Stokes equations in the SIBM, the two-step Runge-Kutta scheme is written by

$$
\bar{u}_{i}^{n+1}=(1-\gamma) \bar{u}_{i}^{n}+\gamma \bar{u}_{i}^{n-1}+\Delta t\left[\alpha\left(\frac{\partial \bar{F}_{i j}^{n}}{\partial x_{j}}+\bar{G}_{i}^{n}\right)+\beta\left(\frac{\partial \bar{F}_{i j}^{n-1}}{\partial x_{j}}+\bar{G}_{i}^{n-1}\right)\right],
$$

where $\alpha, \beta$ and $\gamma$ are the paramaters of two-step Runge-Kutta scheme. In this paper, the parameters are set as $\alpha=11 / 8$, $\beta=-5 / 8$ and $\gamma=-1 / 4$ in order to satisfy the second order of time accuracy. The fractional step approach based on the two-step Runge-Kutta scheme can be writen by

$$
\begin{aligned}
& \bar{u}_{i}^{*}=(1-\gamma) \bar{u}_{i}^{n}+\gamma \bar{u}_{i}^{n-1}+\Delta t\left[\alpha \frac{\partial \bar{F}_{i j}^{\prime n}}{\partial x_{j}}+\beta\left(\frac{\partial \bar{F}_{i j}^{n-1}}{\partial x_{j}}+\bar{G}_{i}^{n-1}\right)\right], \\
& \bar{u}_{i}^{n+1}=\bar{u}_{i}^{*}+\Delta t \alpha\left(-\frac{\partial P^{n}}{\partial x_{i}}+\bar{G}_{i}^{n}\right), \\
& \bar{F}_{i j}^{\prime}=-\bar{u}_{i} \bar{u}_{j}+\left(\frac{1}{R e}+v_{S G S}\right)\left(\frac{\partial \bar{u}_{i}}{\partial x_{j}}+\frac{\partial \bar{u}_{j}}{\partial x_{i}}\right),
\end{aligned}
$$

where $\bar{u}_{i}^{*}$ denotes the fractional step velocity. The resulting pressure equation is solved by the BiCGSTAB method. The convergence criterion of the pressure equation is set as $P_{L_{2}}<1.0 \times 10^{-6}$, where $P_{L_{2}}$ is the $L_{2}$ residual of the pressure. Then, the conservation of mass is satisfied in the range of convergence criterion of the pressure equation. 


\section{LES of turbulent plane channel flow}

In order to validate the present SIBM, the turbulent plane channel flow which is one of the most fundamental wall turbulent flow is considered. In this paper, three LESs are performed, i.e., case-BC, case-IBM, and case-SIBM. In the case-BC, the non-slip boundary condition is imposed on the wall surface. In the case-IBM and case-SIBM, the wall surface is expressed by the virtual boundary and the original IBM and the present SIBM are applied, respectively. The computational domain is shown in Fig. 2. In this figure, $h$ denotes a channel half-width. In the case-IBM and case-SIBM, the wall has the thickness $(0.5 h)$ and the governing equations are solved not only in the channel but also inside the wall.

As shown in Table 1 , the grid resolution is $64 \times 128 \times 64$ points in the $(x, y, z)$ directions, respectively in the flow field. The grid resolution is decided as $\Delta x^{+}=17.67, \Delta y^{+}=2.81$ and $\Delta z^{+}=8.84$ with uniform grid system. In the case-IBM and case-SIBM, inside the wall, the uniform grids of the same resolution as flow field are arranged. The Reynolds number defined by the friction velocity is $R e_{\tau}=180$. The time increment is set as $\Delta t=2.5 \times 10^{-4}$. On the boundary of $x$ and $z$ directions, the velocity and the pressure are imposed by the periodic condition. On the boundary of $y$ direction (the wall boundary), the velocity is imposed by the non-slip condition and the pressure is imposed by the Neumann condition obtained by the normal momentum equation. Also, the velocity condition inside the wall is imposed by the non-slip condition.

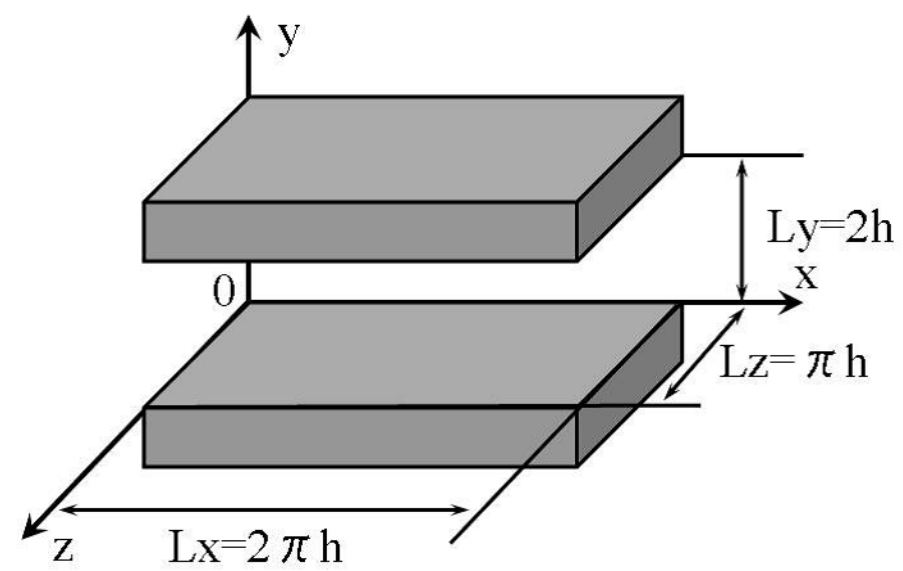

Fig. 2 Computational domain.

\begin{tabular}{c|c|c|c}
\multicolumn{5}{c}{ Table 1 } & Computational conditions. \\
\hline & Presents & Kajishima et al. (LES) & Moser et al. (DNS) \\
\hline$R e_{\tau}$ & 180 & 180 & 180 \\
\hline$N_{x} \times N_{y} \times N_{z}$ & $64 \times 128 \times 64$ & $32 \times 64 \times 32$ & $128 \times 129 \times 128$ \\
\hline$L_{x} \times L_{y} \times L_{z}$ & $2 \pi h \times 2 h \times \pi h$ & $6.4 h \times 2 h \times 3.2 h$ & $4 \pi h \times 2 h \times 1.3 \pi h$ \\
\hline$\Delta x^{+}$ & 17.7 & 36.0 & 17.7 \\
\hline$\Delta y^{+}$ & 2.81 & $1.12 \sim 10.8$ & $0.16 \sim 4.4$ \\
\hline$\Delta z^{+}$ & 8.8 & 18.0 & 5.9 \\
\hline
\end{tabular}

The initial velocity inside the channel is determined by the following Eqs. (16)-(19). These initial velocities are constructed by the trigonometric function, and the averaged velocity defined by the wall law and the logarithmic law, $u_{\text {ave }}$, is added in the flow direction.

$$
\begin{aligned}
& u=2 \pi \cos \left(\frac{4 \pi x}{L_{x}}\right) \sin \left(\frac{2 \pi y}{L_{y}}\right) \sin \left(\frac{4 \pi z}{L_{z}}\right)+\pi \cos \left(\frac{10 \pi x}{L_{x}}\right) \sin \left(\frac{14 \pi y}{L_{y}}\right) \sin \left(\frac{10 \pi z}{L_{z}}\right)+u_{\text {ave }}, \\
& v=2 \sin \left(\frac{4 \pi x}{L_{x}}\right)\left\{1-\cos \left(\frac{2 \pi y}{L_{y}}\right)\right\} \sin \left(\frac{4 \pi z}{L_{z}}\right)-\sin \left(\frac{10 \pi x}{L_{x}}\right)\left\{1-\cos \left(\frac{14 \pi y}{L_{y}}\right)\right\} \sin \left(\frac{10 \pi z}{L_{z}}\right), \\
& w=-\frac{1}{2} \pi \sin \left(\frac{4 \pi x}{L_{x}}\right) \sin \left(\frac{2 \pi y}{L_{y}}\right) \cos \left(\frac{4 \pi z}{L_{z}}\right)-\frac{6}{5} \pi \sin \left(\frac{10 \pi x}{L_{x}}\right) \sin \left(\frac{14 \pi y}{L_{y}}\right) \cos \left(\frac{10 \pi z}{L_{z}}\right),
\end{aligned}
$$




$$
u_{\text {ave }}=\left\{\begin{array}{ll}
y^{+} \quad\left(0 \leq y^{+}<5\right) \\
5 \ln \left(y^{+} / 5\right)+5 \quad\left(5 \leq y^{+}<30\right) \\
2.5 \ln y^{+}+5.5 \quad\left(30 \leq y^{+}<h^{+}\right)
\end{array} .\right.
$$

Figure 3 shows the time-averaged mean velocity of $x$ component and the time-averaged RMS of the turbulence intensity of each components at GS with the reference DNS (Moser, et al., 1999) and LES (Kajishima, et al., 1997) results. These quantitative values are averaged between $t=15$ and 50. In case-BC and case-SIBM, all the time-averaged quantitative values are closer to the DNS results than the LES results. The time-averaged quantitative values of case-BC and case-SIBM are in good agreement with each other. On the other hand, in case-IBM, there are greatly discrepancy with the reference DNS and LES results. In this case, the forcing term is added only near the virtual boundary, so that the pressure jump is formed at the wall boundary. Then, the unphysical pressure oscillations are appeared in the normal direction of the wall (Nishida and Sasao, 2006). As a result, the unphysical velocity oscillations are observed in the normal direction of the wall. This is the essential property of the original IBM. This tendency is similarly observed in the low grid resolution $\left(\Delta y^{+}=5.63\right)$. Therefore, it is found that the present SIBM gives the results equivalent to the conventional ones with wall boundary condition.

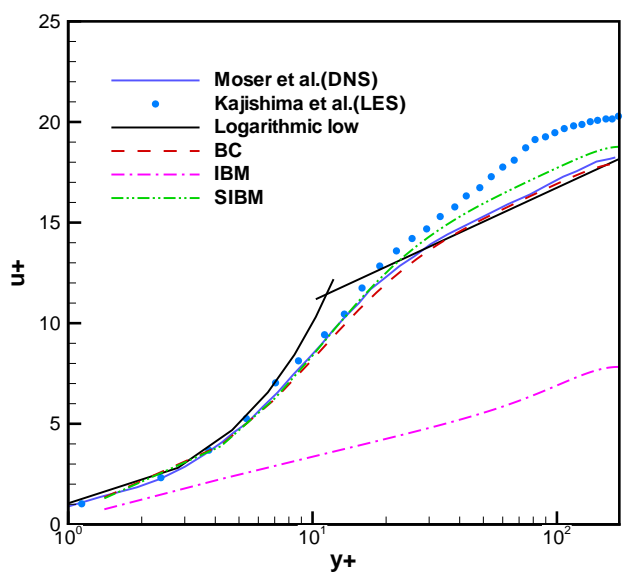

(a) Mean velocity.

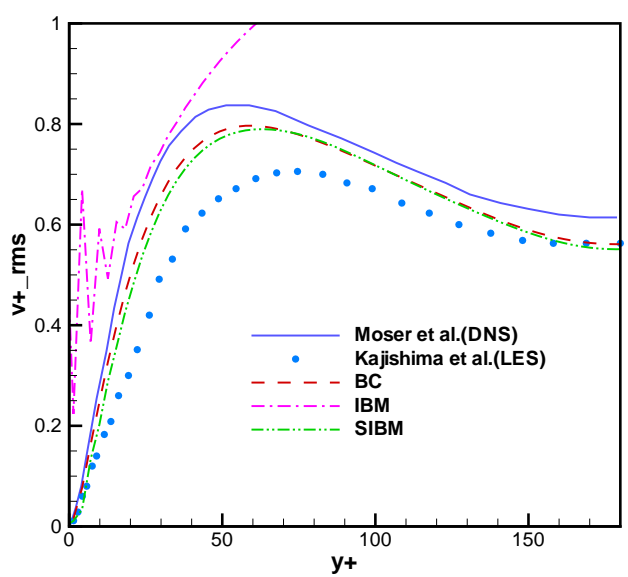

(c) Turblence intensity of $v^{+}$.

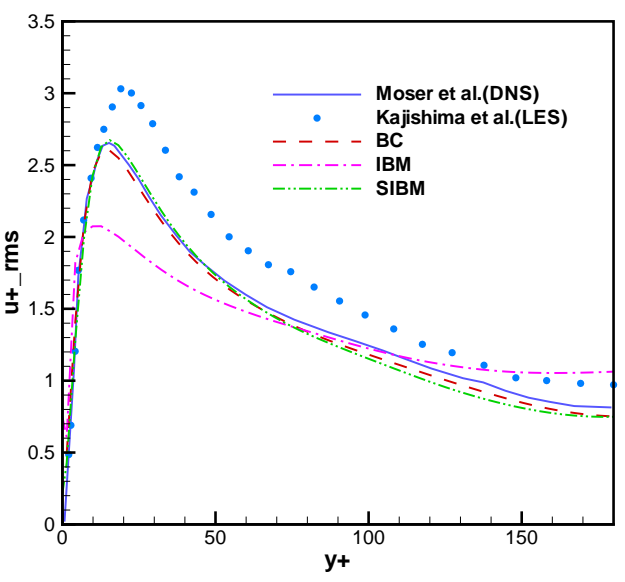

(b) Turblence intensity of $u^{+}$

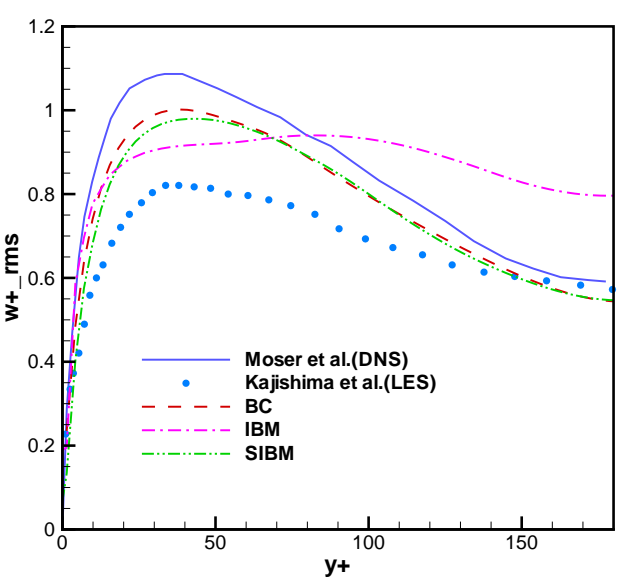

(d) Turblence intensity of $w^{+}$.

Fig. 3 Time averaged flow field in a plane channel by LES at $R e_{\tau}=180$.

\section{LES of flow around a sphere}

In order to validate the present SIBM for the curvilinear boundary, the turbulent flow around a sphere is considered. In this paper, two LESs are performed, i.e., case-IBM, and case-SIBM. The LES of IBM is started from the result of the present SIBM at $t=290$. The computational domain is shown in Fig. 4. $D$ denotes a diameter of the sphere. The 
computational grid is the hierarchical Cartesian grid with 6 levels. The grid resolution is $0.0125 D$ at near the sphere surface. The impulsive start determined by the uniform flow $(u=1, v=0, w=0)$ is adopted. On the inflow boundary, the velocity is fixed by the uniform flow and the pressure is imposed by the Neumann condition obtained by the normal momentum equation. The velocity is extrapolated from the inner points and the pressure is obtained by the Sommerfeld radiation condition (Kawakami, et al., 1994) on the outflow and side boundaries. On the virtual boundary, i.e., the sphere surface, and inside the boundary, the non-slip $(u=0, v=0, w=0)$ condition is imposed. The Reynolds number is set as $R e=3700$ and 10000 in which the flow around a sphere is the turbulent flow. The time increment is set as $\Delta t=0.001$.

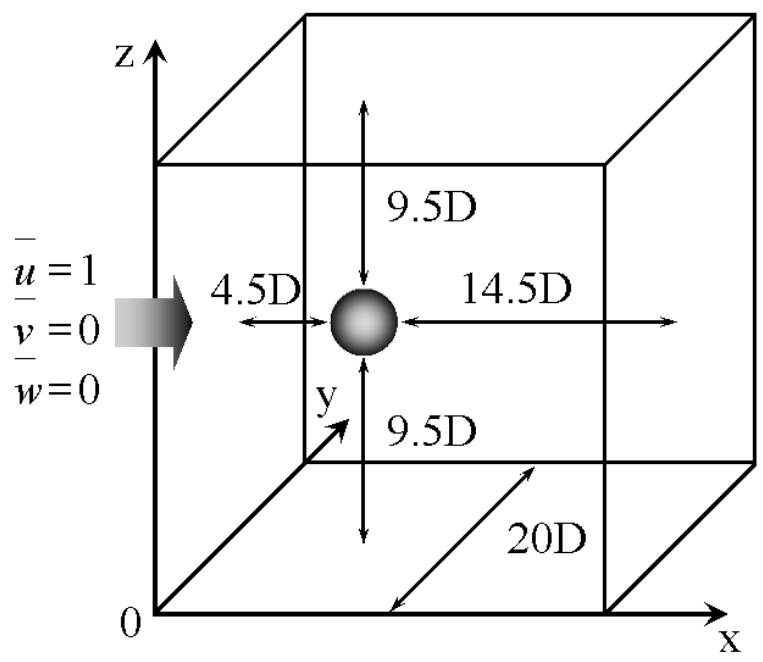

Fig. 4 Computational domain.

Figure 5 shows the second invariant of velocity gradient tensor $\left(Q^{\prime}=0.1\right)$. The vortices are formed at the rear of the sphere and are collapsed at downstream. Moreover, the more vortices are formed at $R e=10000$ than at $R e=3700$.

The present time-averaged drag and lift coefficients are shown with the reference result (Yun, et al., 2006) in Table 2. The present time-averaged drag and lift coefficients are averaged between $t=300$ and 400 . In this paper, the drag and lift coefficients are estimated by two ways, i.e. ,

$$
C_{D}, C_{L_{y}}, C_{L_{z}}=\frac{-\int_{O}\left(\bar{G}_{i}-\bar{u}_{j} \frac{\partial \bar{u}_{i}}{\partial x_{j}}-\frac{\partial \bar{u}_{i}}{\partial t}\right) d s}{\frac{1}{2} \rho_{0} U_{0}^{2} S},
$$

where $S$ and $O$ denote the frontal projected area and the region added forcing term (shown in Fig. 1), and

$$
C_{D}, C_{L_{y}}, C_{L_{z}}=\frac{\int_{b} p_{i} d s+\int_{b} \tau_{i} d s}{\frac{1}{2} \rho_{0} U_{0}^{2} S},
$$

where $b$ denotes the boundary, $p_{i}$ and $\tau_{i}$ denote the interpolated pressure and shear stress on the sphere surface. And, $\rho_{0}$ and $U_{0}$ denote the reference density and velocity of the flow. The conventional estimation, Eq. (21), needs the additional efforts, i.e., the additional grid generation near the boundary and interpolation to the boundary. Conversely, the estimation by using Eq. (20) does not need these additional efforts. In Table 2, it is found that the present SIBM results estimated by two ways are in good agreement with each other. On the other hand, the original IBM results show the different values. Moreover, in the case with $R e=10000$ the original IBM is not stable under the same computational conditions as the SIBM. The present SIBM results with the static SGS model are in very good agreement with the reference results with the dynamic SGS model at both cases of $R e=3700$ and 10000. Figure 6 shows the time history of time-averaged lift coefficients of case-SIBM by Eq. (20). For example, the time-averaged $C_{L_{y}}$ and $C_{L_{z}}$ at $t=300$ are estimated by averaging between $t=0$ and 300. At $R e=3700$, the time-averaged $C_{L_{y}}$ and $C_{L_{z}}$ are approaching to zero with oscillations after $t=300$. Also in $R e=10000$, the same tendency is observed after $t=200$. Yun et al. (Yun, et al., 2006) reported that the values of time-averaged $C_{L_{y}}$ and $C_{L_{z}}$ asymptotically approach to zero when the interval of time average is very long. So, it is predicted that the present LES shows the asymptotic values to zero in the long interval of time average.

As a result, it is found that the present SIBM has the properties which the drag and lift are estimated more directly without the additional efforts, i.e., the additional grid generation near the boundary and the interpolation to the boundary, and the resulting values are in good agreement with the reference ones. 


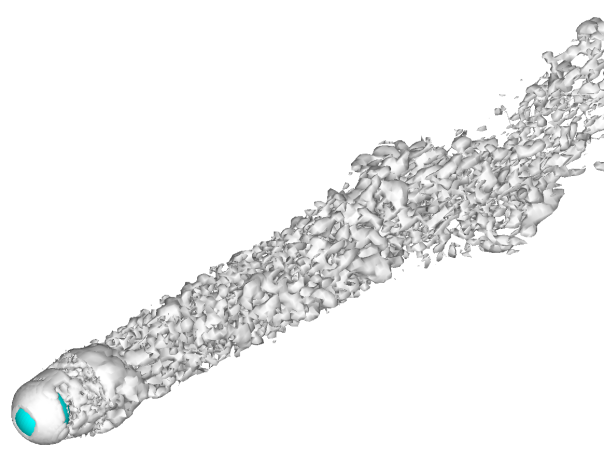

(a) $R e=3700$.

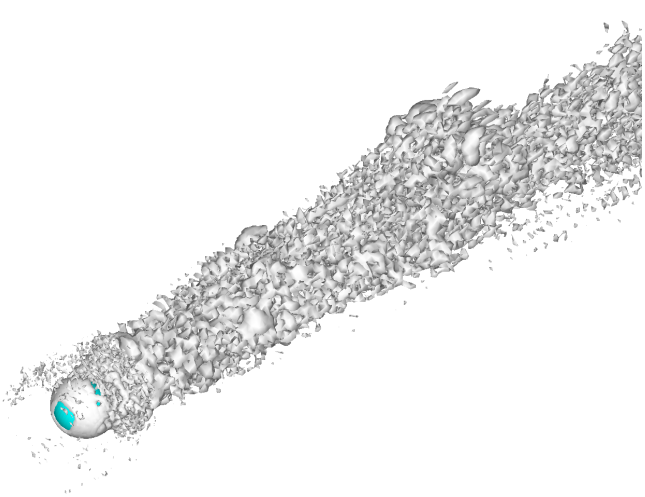

(b) $R e=10000$.

Fig. 5 Second invariant of velocity gradient tensor $Q^{\prime}=0.1$.

Table 2 Comparison of time-averaged drag and lift coefficients.

\begin{tabular}{|c|c|c|c|c|c|c|}
\hline & \multicolumn{3}{|c|}{$\operatorname{Re}=3700}$. & \multicolumn{3}{c|}{$\operatorname{Re}=10000}$. \\
& $\overline{C_{D}}$ & $\overline{C_{L_{y}}}$ & $\overline{C_{L_{z}}}$ & $\overline{C_{D}}$ & $\overline{C_{L_{y}}}$ & ${\overline{C_{L_{z}}}}^{-}$ \\
\hline SIBM-Eq. (20) & 0.37 & $1.4 \times 10^{-2}$ & $1.0 \times 10^{-2}$ & 0.40 & $-1.7 \times 10^{-2}$ & $-4.0 \times 10^{-2}$ \\
\hline SIBM-Eq. (21) & 0.35 & $1.4 \times 10^{-2}$ & $1.0 \times 10^{-2}$ & 0.40 & $-1.6 \times 10^{-2}$ & $-3.9 \times 10^{-2}$ \\
\hline IBM-Eq. (20) & 0.67 & $7.5 \times 10^{-5}$ & $-6.3 \times 10^{-5}$ & - & - & - \\
\hline IBM-Eq. (21) & 0.56 & $8.0 \times 10^{-5}$ & $-5.6 \times 10^{-5}$ & - & - & - \\
\hline Yun et al. & 0.36 & 0 & 0 & 0.39 & 0 & 0 \\
\hline
\end{tabular}

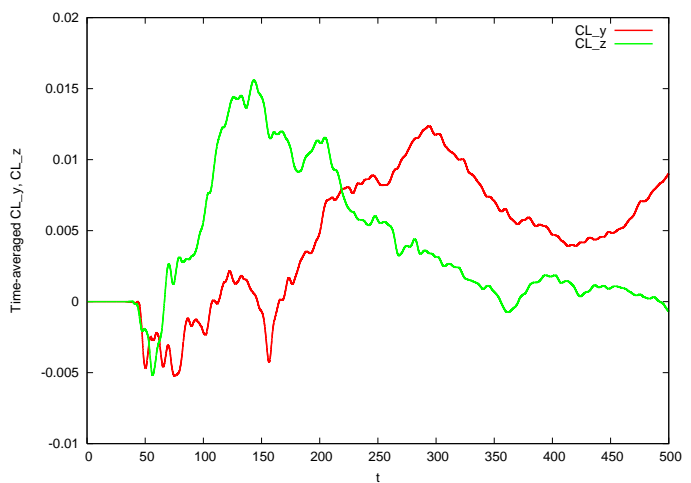

(a) $R e=3700$ by Eq. (20).

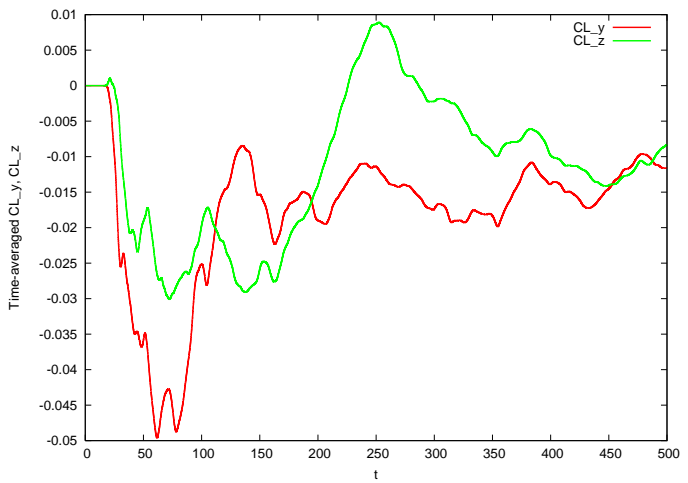

(b) $R e=10000$ by Eq. (20).

Fig. 6 Time history of time-averaged lift coefficients by SIBM.

\section{Concluding Remarks}

In this paper, the SIBM is applied to LES with the static Smagorinsky model. In order to discuss the property for LES, the turbulent plane channel flow and the turbulent flow around a sphere are considered. In the turbulent plane channel flow, the present SIBM results are in good agreement with the results of conventional approach with wall boundary condition and close to the DNS data. In the turbulent flow around a sphere, the present approach gives directly the drag and lift without the additional efforts. Also, the resulting drag and lift are in excellent agreement with the reference ones with the dynamic SGS model. Then, it is concluded that the present SIBM is very promising for the LES of incompressible turbulent flows. 


\section{References}

Fadlun, E. A., Verzicco, R., Orlandi, P. and Mohd-Yosof, J., Combined immersed-boundary finite-difference methods for three-dimensional complex simulations, Journal of Computational Physics, Vol.161 (2000), pp.35-60.

Goldstein, D., Handler, R. and Sirovich, L., Modeling a no-slip flow boundary with an external force field, Journal of Computational Physics, Vol.105 (1993), pp.354-366.

Kajishima, T., Ohta, T., Okazaki, K. and Miyake, Y., High-order finite-difference method for incompressible flows using collocated grid system, Transactions of the Japan Society of Mechanical Engineers, Series B, Vol.63, No.614 (1997), pp.3247-3254 (in Japanese).

Kawakami, K., Nishida, H. and Satofuka, N., An open boundary condition for the numerical analysis of unsteady incompressible flow using the vorticity-streamfunction formulation, Transactions of the Japan Society of Mechanical Engineers, Series B, Vol.60, No.574 (1994), pp.1891-1896 (in Japanese).

Morinishi, Y., Lund, T.S., Vasilyev, O.V. and Moin, P., Fully conservative higher order finite difference schemes for incompressible flow, Journal of Computational Physics, Vol.143 (1998), pp.90-124.

Moser, R., Kim, J. and Mansour, N., Direct numerical simulation of turbulent channel flow up to $R e_{\tau}=590$, Physics of Fluids, Vol.11 (1999), pp.493-495.

Nishida, H. and Sasao, K., Incompressible flow simulations using virtual boundary method with new direct forcing terms estimation, Proceedings of International Conference on Computational Fluid Dynamics 2006(Springer) (2006), pp.185-186.

Nishida, H. and Tajiri, K., Numerical simulation of incompressible flows around a fish model at low Reynolds number using seamless virtual boundary method, Journal of Fluid Science and Technology, Vol.4, No.3 (2009), pp.500-511.

Nishida, H. and Tajiri, K.,Seamless virtual boundary method for incompressible flow simulation with heat transfer, Transactions of the Japan Society of Mechanical Engineers, Series B, Vol.76, No.765 (2010), pp.741-746 (in Japanese).

Peskin, C.S. and McQueen, D.M., A three-dimensional computational method for blood flow in the heart I. Immersed elastic fibers in a viscous incompressible fluid, Journal of Computational Physics, Vol.81 (1989), pp.372-405.

Renaut, R.A., Two-step Runge-Kutta method and hyperbolic partial differential equation, Mathematics of Computation, Vol.55, No.192 (1990), pp.563-579.

Rhie, C.H. and Chow, W.L., Numerical study of the turbulent flow past an airfoil with trailing edge separation, AIAA Journal, Vol.21, No.11 (1983), pp.1525-1532.

Saiki, E.M. and Biringen, S., Numerical simulation of a cylinder in uniform flow: Application of a virtual boundary method, Journal of Computational Physics, Vol.123 (1996), pp.450-465.

Tajiri, K., Nishida, H. and Tanaka, M., Numerical simulation of incompressible flows with heat transfer using seamless immersed boundary method, Journal of Computational Science and Technology, Vol.7, No.2 (2013), pp.286-296.

Yun, G., Kim, D. and Choi, H., Vortical structures behind a sphere at subcritical Reynolds numbers, Physics of Fluids, Vol.18 (2006), 015102 